\title{
Adolescent multiple risk behaviours cluster by number of risks rather than distinct risk profiles in the ALSPAC cohort
}

\author{
Caroline Wright ${ }^{*}$ (D), Jon Heron, Rona Campbell, Matthew Hickman and Ruth R. Kipping
}

\begin{abstract}
Background: Experimentation with new behaviours during adolescence is normal. However, engagement in two or more risk behaviours, termed multiple risk behaviours is associated with socioeconomic disadvantage and poor health and social outcomes. Evidence of how adolescents cluster based on their risk behaviours is mixed.

Methods: Latent Class Analysis was used to study patterns of engagement in 10 self-reported risk behaviours (including substance use, self-harm and sexual health) from the Avon Longitudinal Study of Parents and Children (ALSPAC) cohort at ages 15-16 years. Data was available for 6556 adolescents. Associations between risk profile and sex were explored.

Results: A 3-class model for both females and males was deemed to have acceptable fit. Whilst we found evidence of a sex difference in the risk behaviours reported within each class, the sex-specific results were very similar in many respects. For instance, the prevalence of membership of the high-risk class was $8.5 \%$ for males and $8.7 \%$ for females and both groups had an average of 5.9 behaviours. However, the classes were both statistically dubious, with class separation (entropy) being poor as well as conceptually problematic, because the resulting classes did not provide distinct profiles and varied only by quantity of risk-behaviours.

Conclusion: Clusters of adolescents were not characterised by distinct risk behaviour profiles, and provide no additional insight for intervention strategies. Given this is a more complicated, software-specific method, we conclude that an equally effective, but more readily replicable approach is to use a count of the number of risk behaviours.
\end{abstract}

Keywords: ALSPAC, Multiple risk behaviours, Public health intervention, Latent class analysis, Clustering

\section{Background}

There is growing interest in addressing adolescent multiple risk behaviours (MRB) [1]. MRB is broadly defined as engagement in two or more risk behaviours [2]. Many modifiable MRB (smoking, excessive alcohol intake, poor diet) originate in adolescence, but may become habitual in adulthood, thereby increasing risk of comorbidities and premature mortality. Studies have shown that adolescents involved in one risk behaviour are more likely to be involved in others [3-5]. This can apply both between substances (tobacco, alcohol and illicit drugs) and

\footnotetext{
* Correspondence: caroline.wright@bristol.ac.uk

Population Health Sciences, Bristol Medical School, Barley House, University of Bristol, Oakfield Grove, Clifton, Bristol BS8 2BN, UK
}

between substance use and other behaviours such as sexual risk, self-harm and antisocial behaviour [5].

It has been hypothesised that interventions targeting one behaviour are less successful because they do not address co-occurring MRB. Evidence indicates that universal school-based interventions targeting MRB are most efficacious in preventing tobacco smoking, alcohol consumption, illicit drug use, antisocial behaviour and increasing physical activity among young people. Evidence was less conclusive for cannabis use, sexual risk and unhealthy diet [2].

Research considering a wider range of behaviours from multiple domains to inform the scope of interventions is complex, often with strongly related behaviours. Hence data-reduction techniques are adopted, to render the

(c) The Author(s). 2020 Open Access This article is distributed under the terms of the Creative Commons Attribution 4.0 International License (http://creativecommons.org/licenses/by/4.0/), which permits unrestricted use, distribution, and reproduction in any medium, provided you give appropriate credit to the original author(s) and the source, provide a link to the Creative Commons license, and indicate if changes were made. The Creative Commons Public Domain Dedication waiver (http://creativecommons.org/publicdomain/zero/1.0/) applies to the data made available in this article, unless otherwise stated. 
information more manageable. Approaches can be classified as either person-centred or variable-centred. There is often a mathematical equivalence between opposing methods, e.g. Confirmatory Factor Analysis (CFA) and Latent Class Analysis (LCA), the choice of model therefore cannot be motivated solely by data. In public health research, LCA brings the potential to extract and study individuals with differing profiles of behaviour who might respond differently to a targeted intervention [6]. Variable-centred methods include Principle Component Analysis (PCA) and Confirmatory/Exploratory Factor Analysis (CFA/EFA) which simplify observed (co) variation into behavioural traits [7], whilst alternative approaches cluster the behaviours into smaller subsets.

Beginning with variable-centred studies, multiple group CFA was used by de Looze et al. to examine clustering of smoking, drunkenness and cannabis use and early sexual activity among adolescents aged $\sim 15$ years, across 27 European and North American countries. They found that substance use and early sexual activity loaded on a single underlying cluster consistently across countries [1]. Unfortunately, because they have not considered a wider range of MRB, there may be other cooccurring MRB which we are not aware of. A Dutch study used EFA and CFA to investigate whether a wide range of health and antisocial behaviours clustered. Several separate but interrelated clusters were found. At age $12-15$ years one broad cluster and a second cluster comprising alcohol, tobacco smoking and drug use. At age 16-18 years alcohol, unsafe sex, unlawful traffic behaviour and vigorous physical activity; and a second cluster of aggressive behaviour, tobacco smoking, drug use, little sleep and delinquency [8]. It seems that these age groupings have been imposed on the data analysis (rather than them naturally clustering in these groups), so it is difficult to know whether and how age would have impacted the kinds of clusters identified. Hierarchical Agglomerative Cluster Analysis (HACA), was used to explore MRB in Saudi Arabian males, aged $\sim 13-19$ years. There was evidence for a non-adherence to prevention group (low fruit consumption, less frequent tooth brushing and low physical activity) and a risk behaviour group (high sweets' consumption, smoking and physical fighting), regardless of age [7]. This analysis is limited by both the small number and type of risk considered as well as the focus on only male adolescents. HACA was also adopted in a study exploring clustering of 17 risk behaviours among Brazilian adolescents aged 13-15 years [9]. This also generated a lack of adherence to preventive behaviours (less frequent hygiene practices, unprotected sex, skipping breakfast, no dental visits), and undertaking risky conduct (current smoking, illegal drug use, no helmet and seatbelt use, high sugar intake, physical fighting and current drinking) and a second unhealthy lifestyle group (sedentary habits, such as insufficient physical activity and eating while watching TV or studying, and diet poor in fruit).

Among the person-centred studies, a New Zealand study used LCA to examine clustering of MRB (alcohol use problems, smoking cigarettes, marijuana use, motor vehicle risk, violence, unsafe sexual health, delinquency, depression and attempted suicide) in a national sample of secondary school students, aged $12-18$ years (80\% of the sample were aged $14 / 15$ years). The analysis identified a four-class model: the 'healthy' group which constituted the majority of students (79.6\%), all of whom presented with $\leq 1$ health concerns; the 'distressed' group (5.9\% of the sample) the majority of whom had depressive symptoms, $48 \%$ of whom had attempted suicide in the past year and $52 \%$ of whom presented $\geq 3$ health concerns. The 'risky' group (10.8\% of the sample) with higher rates of risky behaviours, but low rates of emotional concerns. The 'multiple' group (3.5\% of the sample), reported high levels of both risky behaviours and emotional problems [10]. A similar LCA investigating clustering of MRB among Australians aged 18 years [11], found three classes: moderate risk (52\%): moderately likely to binge drink and not eat enough fruit, high probability of insufficient vegetable intake; inactive, nonsmokers (24\%): high probabilities of not meeting guidelines for physical activity, sitting time and fruit/vegetable consumption, very low probability of smoking; and smokers and binge drinkers (24\%): high rates of smoking and binge drinking, poor fruit/vegetable intake. The classes were differentially associated with psychological distress, depression and anxiety. Using LCA, Laxer et al. [12] examined the associations of $15 \mathrm{MRB}$ and overweight/obesity among Canadian adolescents in grades 9 to 12 (age 14-18 years). All groups were more likely to be overweight/obese when compared to the health conscious group: traditional school athletes odds ratio $(\mathrm{OR})=1.15(95 \% \mathrm{CI}: 1.03-1.29)$, inactive screenagers OR = 1.33(95\%CI:1.19-1.48) and moderately active substance users OR $=1.27$ (95\%CI:1.14-1.43).

Evidence regarding the clustering of MRB is mixed, with some research finding distinct risk profiles, while others find only broad clusters. This is further obscured when age and sex/gender are considered. Although most studies presented here cover a range of ages across adolescence, two studies de Looze et al. [1] and Champion et al. [11] focus on age 15 and 18 years, respectively, which may be considered limitations to their analysis. While some studies include wide-ranging behaviours, others continue to use only a limited number or type of risk behaviour. Reducing the analysis to similar MRB that are hypothesised to co-occur, runs the risk of missing important relationships that have not been included. It is therefore imperative to test the validity of these 
profile-specific approaches, incorporating a larger number of divergent risk behaviours for a UK adolescent population.

We aimed to explore the utility of a latent class approach to investigate patterns of MRB using the Avon Longitudinal Study of Parents and Children (ALSPAC) cohort with a view to informing future public health interventions. We chose to examine MRB at age $\sim 16$ years because adolescent brain development, is second only to infancy as a dynamic period, making it a crucial period of study [13]. In addition, the General Certificate of Secondary Education (GCSE) examinations are completed at age 16 in the UK, determining entrance to post-16 education and university, making it a time of great importance. Further, evidence using ALSPAC data shows that, while not at their highest prevalence, age 16 is when both tobacco and cannabis see their most dramatic increase in use [14]. Similarly, alcohol use is rapidly increasing and antisocial behaviour is at its peak at this age [15].

\section{Methods}

\section{Participants}

Data were drawn from ALSPAC, an ongoing prospective population-based study designed to investigate the effects of a wide range of influences on the health and development of children [16, 17]. Pregnant women resident in Avon, UK with expected dates of delivery 1st April 1991 to 31st December 1992 were invited to take part in the study. The initial number of pregnancies enrolled is 14,541 . Of these initial pregnancies, there was a total of 14,676 foetuses, resulting in 14,062 live births and 13,988 children who were alive at 1 year of age. Please note that the study website contains details of all the data that is available through a fully searchable data dictionary and variable search tool (http://www.bristol. ac.uk/alspac/researchers/our-data/). Ethical approval for the study was obtained from the Avon Longitudinal Study of Parents and Children Ethics and Law Committee and local Research Ethics Committees.

\section{Multiple risk behaviour indicators in adolescence}

Data was taken from participants' responses to both: (i) a previously published self-completed questionnaire issued at a clinic attended at age 15 (median age 15 years and 5 months) and, (ii) a previously published postal questionnaire administered at age 16 (median age 16 years and 7 months) (http://www.bristol.ac.uk/media-library/sites/alspac/documents/questionnaires/CCS-lifeof-a-16-plus-teenager.pdf) (see Supplementary Table 1). In previous analyses we have included unprotected sexual intercourse as a thirteenth MRB $[18,19]$. However, this was excluded due to its dependency on early sexual behaviour and to avoid structural zeros in the statistical analysis.

\section{Statistical methods Simple bivariate analysis}

Polychoric correlations were derived for each pair of MRB (see Supplementary Table 2). Two behaviours (physical inactivity and TV viewing) were weakly correlated with the other behaviours and each other $(\leq 0.18)$. We therefore dropped them from our subsequent analysis as they would only diminish model fit.

\section{Latent class analyses}

The remaining MRB were subjected to a succession of latent class analyses (LCA). LCA assumes that observed associations between variables, are due to a categorical latent variable with two or more classes. Additional classes were added incrementally until the resulting model was deemed acceptable based on a range of statistical and substantive criteria. Analyses were performed separately for females and males because there were some sex differences in the prevalence of MRB (see Supplementary Figure 1).

The Bayesian Information Criterion (BIC) [20] is the most commonly-used fit statistic for comparing LCA models. A function of both the likelihood and number of estimated parameters, the BIC penalises model complexity. Using this statistic, the model with the lowest BIC would be deemed satisfactory.

Conditional independence is an assessment of the remaining association between each pair of measurements once the effect of the latent class variable has been removed. There is currently no accepted threshold for this measure. In addition to a global measure of fit, the individual standardized residuals can be examined to indicate specific areas of poor fit.

The Bootstrap Likelihood Ratio Test (BLRT) and the Lo-Mendell-Rubin (LMR) test statistics [21] both assess change in model fit when adding an additional class. Unlike the LMR, the BLRT makes no distributional assumptions and simulation work has so far shown this measure to be superior [6], however the BLRT can be conservative and may reject all the models considered.

LCA modelling produces a class-assignment probability which describes the confidence each participant can be assigned to each latent class. Entropy, also referred to as classification accuracy, summarises this information as a single measure ranging from zero to one (one indicating no uncertainty). Entropy is of little use in determining the optimal model [22] and can be poor in simulation studies even when the correct model is estimated [23].

Whilst LCA has been promoted as a method to facilitate targeted public health interventions [6] we propose that this is highly dependent on clearly defined, well- 
separated groups of individuals. Consequently, we regard entropy as an indicator of model utility since if it is low and individuals are poorly classified then the resulting classification is of little use as a targeting tool in future interventions.

Analysts often place a limit on the size of the resulting classes. This pragmatic decision is to facilitate any planned future studies or use of LCA. Here we only considered models where all classes contained at least 50 participants.

Both concurrent and face validity are important criteria with which to assess and compare these models. As LCA is an exploratory technique it is important that the within-class profiles are consistent with available evidence. The extraction of implausible classes can be used to justify one model over another. Further, classes which appear similar and cannot be distinguished in relation to any association with key predictors may be of little subtantive use.

\section{Missing data}

When estimating models with multiple dependent variables, a maximum likelihood (ML) based approach can be employed to address the problem of partial nonresponse as an alternative to Multiple Imputation (MI), which is based on the missing at random (MAR) assumption i.e. that any differences between the missing and observed values, can be explained by differences in the observed data [24].

LCA permits the inclusion of respondents with $\geq 1$ MRB. To assess the impact of including partially complete data, the same models were estimated on three different subsamples, 1: complete case, 2 : all participants with $\leq 4$ missing values and 3: all available data. For the main text of this manuscript we focus on sample 2. Supplementary figures 2 and 3 show comparisons across the three samples.

\section{Results}

\section{Analysis sample}

Ten thousand seven hundred fifty-nine participants were invited to contribute to both a clinic-based $(n=9979)$ and postal questionnaire $(n=9510)$ data collection. Of these, $6556(61 \%)($ male $=2965 /$ female $=3591)$ provided some information on MRB. Four thousand eight hundred thirty young people $($ male $=1981 /$ female $=2849)$ were missing at most four responses and 2930 (male = $1195 /$ female $=1735)$ had complete data.

\section{Latent class models}

There was good support for a 3-class model for females and some support for both a 3- and 4-class model for males (model fit statistics are in Supplementary Table 3). Inspection of standardized bivariate residuals for the male and female 3- and 4-class models (Supplementary Figures 4 \& 5) showed acceptably low numbers of large residuals for the more parsimonious models. Further, the fourth class was small and made little theoretical sense. Consequently, the 3-class model was chosen for both sexes. We note however that class separation was poor for all models, with entropy typically $0.60-0.70$ (entropy $>0.80$ is considered good, $>0.90$ is considered excellent [25]).

\section{Within-class profiles of risk behaviour}

The sex-specific class sizes and average number of MRB reported within each class are shown in Table 1. For males there was a low risk' group (51.6\%) characterised by few MRB other than a low-to-moderate level of criminality/ antisocial behaviour (ASB), alcohol use and lack of helmet wearing, with 1.0 MRB exhibited on average. 'Medium risk' (40.0\%): characterised by a high probability of criminality/ASB and alcohol use and moderate likelihood of road/injury risks, with 3.3 average MRB. 'High risk' (8.5\%): similar to medium risk but with the addition of increased chance of tobacco-use and cannabis/other illict drug use, with 5.9 average MRB (see Fig. 1).

For females there was also a low risk' group (52.3\%): characterised by few MRB although there were subtle differences with females associated with lower probability of criminality/ASB, scooter risk and helmet risk than males, but a slightly higher probablity of self-harm and sex before age 16 years, with 0.8 MRB exhibited on

Table 1 Average number of MRBs reported within each class

\begin{tabular}{|c|c|c|c|c|c|c|}
\hline & \multicolumn{2}{|c|}{ Sample 1: Complete data } & \multicolumn{2}{|c|}{ Sample 2: $\leq 4$ missing values } & \multicolumn{2}{|c|}{ Sample 3: All available data } \\
\hline & Prevalence & Average \# MRB & Prevalence & Average \# MRB & Prevalence & Average \# MRB \\
\hline Male & $(n=1195)$ & & $(n=1981)$ & & $(n=2965)$ & \\
\hline Class 1 (low risk) & $58.3 \%$ & 1.2 & $51.6 \%$ & 1.0 & $54.2 \%$ & 1.1 \\
\hline Class 2 (medium risk) & $31.8 \%$ & 3.4 & $40.0 \%$ & 3.3 & $36.3 \%$ & 3.3 \\
\hline Class 3 (high risk) & $9.8 \%$ & 5.7 & $8.5 \%$ & 5.9 & $9.6 \%$ & 6.0 \\
\hline Female & $(n=1735)$ & & $(n=2849)$ & & $(n=3591)$ & \\
\hline Class 1 (low risk) & $58.4 \%$ & 0.9 & $52.3 \%$ & 0.8 & $50.3 \%$ & 0.8 \\
\hline Class 2 (medium risk) & $34.8 \%$ & 3.1 & $38.9 \%$ & 3.0 & $40.3 \%$ & 2.9 \\
\hline Class 3 (high risk) & $6.8 \%$ & 6.2 & $8.7 \%$ & 5.9 & $9.3 \%$ & 6.0 \\
\hline
\end{tabular}




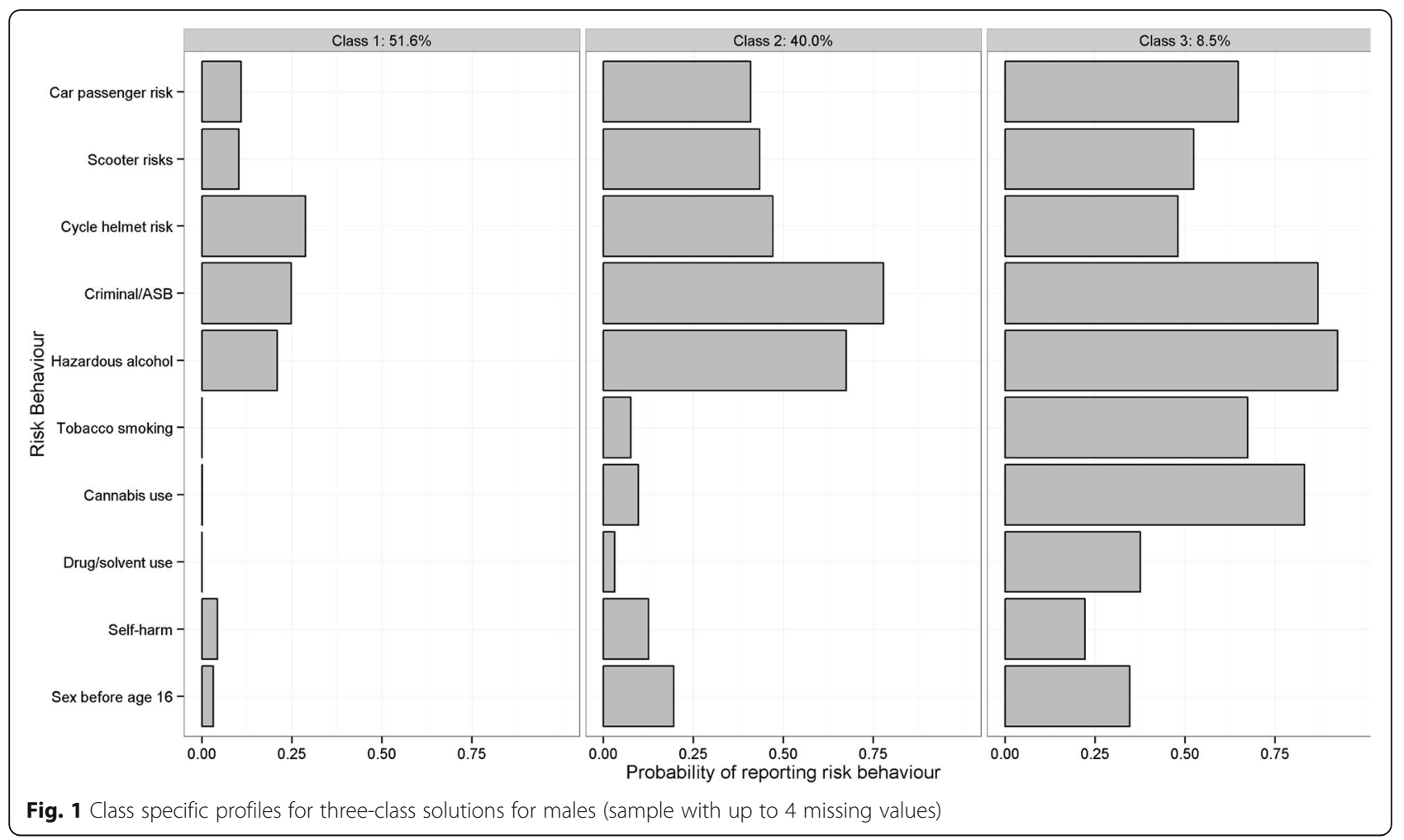

average. Similarly, the 'medium risk'group (38.9\%) was characterized by moderate-to-large probability of criminality/ASB and alcohol use and moderate likelihood of car-passenger risk but not the other road/injury risks.
Self-harm and sex before 16 were also prominent, with 3.0 average MRB. 'High risk' (8.7\%): all behaviours were raised with the exception of scooter-use and helmet wearing, with 5.9 average MRB (see Fig. 2).

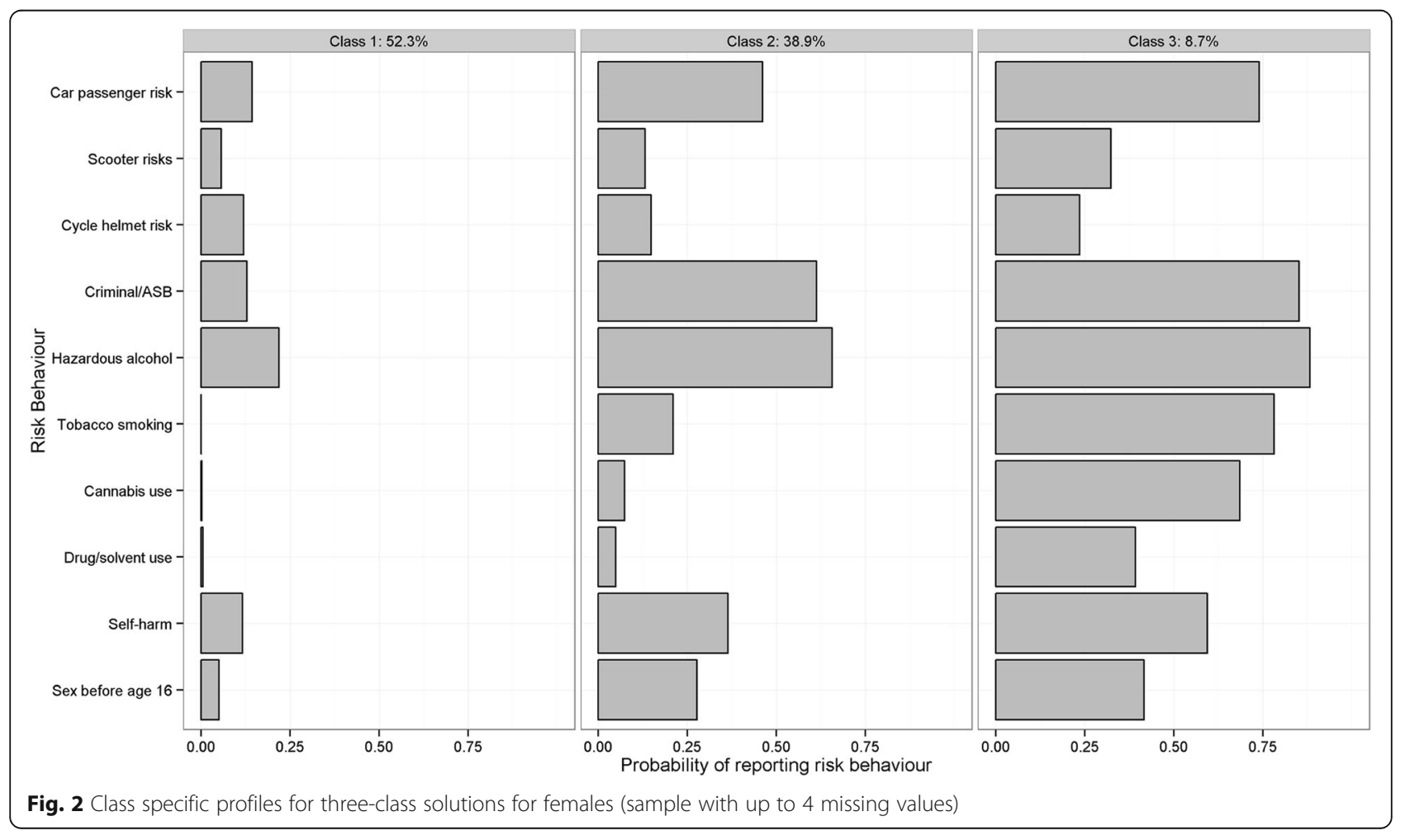




\section{Discussion}

\section{Main findings}

Our LCA found a 3-class model for both females and males and evidence for multiple underlying subgroups. However, classes were not characterised by distinct risk profiles. Rather they varied by quantity of behaviours, with some sex differences in the intensity of these behaviours within classes. The proportion of the cohort in each class and the average number of risk behaviours $(\sim 1, \sim 3, \sim 6)$ for each class respectively, was remarkably consistent for females and males. Likewise, the patterning of MRB within each class was broadly similar but with small differences in intensity for scooter risk behaviours, lack of cyclehelmet wearing, criminalty/ASB, self-harm and earlysex (typically more sex-specific behaviours). In addition to indistinct class profiles, class separation (entropy) was poor. Whilst it is possible for the correct model to yield poor class delineation in this way, poor entropy will limit the extent to which such a classification will inform the design of a targeted public health intervention.

We have demonstrated that the utilty of the three classes as an indicator of behaviours to target for MRB prevention, is poor, given the absence of any clustering of types of risk behaviour. The value of the three latent classes in providing empirical support for, or evidence with which to test theories of MRB is also limited.

\section{Strengths and limitations}

This is the first paper that has conducted LCA of a wide range of adolescent MRB in the UK. We used prospectively collected data for a large sample of adolescents, with a wide range of MRB. Further, our choice of behaviours was informed by discussion with two groups of adolescents through the DECIPHer ALPHA young person's research advisory group (http://decipher.uk.net/public-involvement/youngpeople/). A limitation is that this research only captures MRB at age 16 years, which may impact the conclusions of the analysis and would benefit from a repeated measures analyses of MRB across adolescence to elicit how engagement in MRB clusters across time.

\section{Conclusion}

Our research calls into question the utility of the clustering approach as a useful way to describe patterns of MRB. The three classes identified were mainly distinguished by the number of MRB engaged in. A better strategy, therefore, is to sum the behaviours to create an overall score. We have shown in a previous analysis that despite individual risk behaviours patterning differently according to sex, females and males engaged in a similar number of MRB [26]. Further, while the associations between individual MRB and socioeconomic status were highly variable, a more consistent relationship was established with MRB score [18]. The evidence points to the volume of behaviours being the critical factor, rather than the types of behaviours engaged in. This has implications for the design of public health interventions aimed at reducing $M R B$, providing further evidence that MRB co-occur among adolescents, and therefore prevention strategies should focus on multiple rather than single risk behaviours. This is already being encouraged in national policy and while there is some evidence this is being implemented by local authorities among adults [27], more work needs to be done regarding adolescents. Prevention strategies should focus on the quantity, rather than the type of MRB and evidence has shown that interventions targeting multiple-substance use can also be effective for other MRBs, providing an excellent basis for MRB prevention. A recent Cochrane Systematic Review showed that universal school-based interventions are most effective in preventing alcohol consumption, tobacco use, illicit drug use and antisocial behaviour, and increasing physical activity among young people but did not find strong evidence of benefit for family or individual-level interventions for the MRB studied [2]. Therefore, efforts for MRB prevention should focus on developing appropriate school-based interventions.

\section{Supplementary information}

Supplementary information accompanies this paper at https://doi.org/10. 1186/s12889-020-8369-6.

\begin{abstract}
Additional file 1 : Table S1. Details on the derivation of the risk behaviours. Table S2. Polychoric correlations between 12 risk behaviours (males below and females above main diagonal). Table S3. Model fit statistics for 10-item Latent Class models across three alternative samples. Figure S1. Prevalence of each risk behaviour (complete case sample, $n=$ 1195 males / 1735 females). Figure S2. Class-specific MRB profiles Males. Figure S3. Class-specific MRB profiles - Females. Figure S4. Bivariate residuals for 3 and 4 -class males' models (complete case sample (S1)). Figure S5. Bivariate residuals for 3 and 4-class females' models (complete case sample (S1))
\end{abstract}

Abbreviations

ALSPAC: Avon Longitudinal Study of Parents and Children; LCA: Latent Class Analysis; MRB: Multiple Risk Behaviour

\section{Acknowledgements}

We are extremely grateful to all the families who took part in this study, the midwives for their help in recruiting them, and the whole ALSPAC team, which includes interviewers, computer and laboratory technicians, clerical workers, research scientists, volunteers, managers, receptionists and nurses. This publication is the work of the authors who will serve as guarantors for the contents of this paper. The UK Medical Research Council and Wellcome 
Trust (Grant Ref: 092731) and the University of Bristol provide core support for ALSPAC.

\section{Authors' contributions}

All authors have read and approved the manuscript. JH, RK, RC and $\mathrm{MH}$ devised the design of the study and discussed the analysis. JH undertook the analyses. JH and RK wrote the first draft of the manuscript. CW, RC and $\mathrm{MH}$ edited the paper. All authors read and approved the final manuscript.

\section{Funding}

The work was undertaken with the support of The Centre for the Development and Evaluation of Complex Interventions for Public Health Improvement (DECIPHer), a UKCRC Public Health Research Centre of Excellence. Joint funding (MR/KO232331/1) from the British Heart Foundation, Cancer Research UK, Economic and Social Research Council, Medical Research Council, the Welsh Government and the Wellcome Trust, under the auspices of the UK Clinical Research Collaboration, is gratefully acknowledged. CW is funded by a Cancer Research UK Population Research Postdoctoral Fellowship (C60153/A23895). The UK Medical Research Council and Wellcome (Grant ref.: 102215/2/13/2) and the University of Bristol provide core support for ALSPAC. These funding bodies had no role in the design, analysis or interpretation of the study, or in writing the manuscript. This publication is the work of the authors and they will serve as guarantors for the contents of this paper.

\section{Availability of data and materials}

Please note that the study website contains details of all the data that is available through a fully searchable data dictionary and variable search tool (http://www.bristol.ac.uk/alspac/researchers/our-data/).

\section{Ethics approval and consent to participate}

Ethical approval for the study was obtained from the Avon Longitudinal Study of Parents and Children Ethics and Law Committee and local Research Ethics Committees: Bristol and Weston Health Authority: E1808 Children of the Nineties: Avon Longitudinal Study of Pregnancy and Childhood (ALSPAC). (28th November 1989), Southmead Health Authority: 49/89 Children of the Nineties - "ALSPAC". (5th April 1990), Frenchay Health Authority: 90/8 Children of the Nineties. (28th June 1990), 15 Year Clinic: Central \& South Bristol Research Ethics Committee (UBHT): 06/Q2006/53 Avon Longitudinal Study of Parents and Children (ALSPAC), Hands on Assessments: Teen Focus 3 (Focus 15+). (7th August 2006) (Confirmed 15th September 2006) and 17 Year clinic: North Somerset \& South Bristol Research Ethics Committee: 08/H0106/9 Avon Longitudinal Study of Parents and Children (ALSPAC), Hands on Assessments: Teen Focus 4 (Focus 17+) (18th November 2008). Informed consent for the use of data collected via questionnaires and clinics was obtained from participants following the recommendations of the Avon Longitudinal Study of Parents and Children Ethics and Law Committee at the time.

Study participants who complete questionnaires consent to the use of their data by approved researchers. Up until age 18 an overarching parental consent was used to indicate parents were happy for their child (the study participant) to take part in ALSPAC. Consent for data collection and use was implied via the written completion and return of questionnaires. Study participants have the right to withdraw their consent for specific elements of the study, or from the study as a whole, at any time.

\section{Consent for publication}

N/A.

\section{Competing interests}

The authors declare that they have no competing interests.

Received: 22 August 2019 Accepted: 18 February 2020 Published online: 04 March 2020

\section{References}

1. de Looze M, Ter Bogt TF, Raaijmakers QA, Pickett W, Kuntsche E, Vollebergh WA. Cross-national evidence for the clustering and psychosocial correlates of adolescent risk behaviours in 27 countries. Eur J Pub Health. 2015;25(1): 50-6.
2. MacArthur G, Caldwell DM, Redmore J, Watkins SH, Kipping R, White J, et al. Individual-, family-, and school-level interventions targeting multiple risk behaviours in young people. Cochrane Database Syst Rev. 2018;10: CD009927.

3. Busch V, Van Stel HF, Schrijvers AJ, de Leeuw JR. Clustering of health-related behaviors, health outcomes and demographics in Dutch adolescents: a cross-sectional study. BMC Public Health. 2013;13:1118.

4. Dumith SC, Muniz LC, Tassitano RM, Hallal PC, Menezes AM. Clustering of risk factors for chronic diseases among adolescents from southern Brazil. Prev Med. 2012;54(6):393-6.

5. Poulin C, Graham L. The association between substance use, unplanned sexual intercourse and other sexual behaviours among adolescent students, Addiction. 2001;96(4):607-21.

6. Lanza ST, Rhoades BL. Latent class analysis: an alternative perspective on subgroup analysis in prevention and treatment. Prev Sci. 2013;14(2):157-68.

7. Alzahrani S, Watt R, Sheiham A, Aresu M, Tsakos G. Patterns of clustering of six health-compromising behaviours in Saudi adolescents. BMC Public Health. 2014;14(1):1215.

8. van Nieuwenhuijzen $\mathrm{M}$, et al. Clustering of health-compromising behavior and delinquency in adolescents and adults in the Dutch population. Prev Med. 2009:48(6):572-8.

9. Jordão LMR, Malta DC, Freire MCM. Clustering patterns of oral and general health-risk behaviours in Brazilian adolescents: findings from a national survey. Community Dent Oral Epidemiol. 2018;46(2):194-202.

10. Noel $\mathrm{H}$, et al. Clustering of adolescent health concerns: a latent class analysis of school students in New Zealand. J Paediatr Child Health. 2013; 49(11):935-41.

11. Champion KE, Mather M, Spring B, Kay-Lambkin F, Teesson M, Newton NC. Clustering of Multiple Risk Behaviors Among a Sample of 18-Year-Old Australians and Associations With Mental Health Outcomes: A Latent Class Analysis. Front Public Health. 2018;6:135

12. Laxer RE, Brownson RC, Dubin JA, Cooke M, Chaurasia A, Leatherdale ST. Clustering of risk-related modifiable behaviours and their association with overweight and obesity among a large sample of youth in the COMPASS study. BMC Public Health. 2017;17(1):102.

13. Andersen SL, Teicher MH. Stress, sensitive periods and maturational events in adolescent depression. Trends Neurosci. 2008;31(4):183-91.

14. Jones HJ, Gage SH, Heron J, Hickman M, Lewis G, Munafò MR, et al. Association of Combined Patterns of tobacco and Cannabis use in adolescence with psychotic experiences. JAMA Psychiatry. 2018;75(3):240-6.

15. Hammerton G, Mahedy L, Murray J, Maughan B, Edwards AC, Kendler KS, et al. Effects of excessive alcohol use on antisocial behavior across adolescence and early adulthood. J Am Acad Child Adolesc Psychiatry. 2017;56(10):857-65.

16. Boyd A, Golding J, Macleod J, Lawlor DA, Fraser A, Henderson J, Molloy L, Ness A, Ring S, Davey Smith G. Cohort profile: the 'children of the 90s'-the index offspring of the Avon longitudinal study of parents and children. Int J Epidemiol. 2013;42(1):111-27.

17. Fraser A, Macdonald-Wallis C, Tilling K, Boyd A, Golding J, Davey Smith G, et al. Cohort profile: the Avon longitudinal study of parents and children: ALSPAC mothers cohort. Int J Epidemiol. 2013:42(1):97-110.

18. Kipping RR, Smith M, Heron J, Hickman M, Campbell R. Multiple risk behaviour in adolescence and socio-economic status: findings from a UK birth cohort. Eur J Pub Health. 2015;25(1):44-9.

19. Wright C, Kipping R, Hickman M, Campbell R, Heron J. Effect of multiple risk behaviours in adolescence on educational attainment at age 16 years: a UK birth cohort study. BMJ Open. 2018:8(7):e020182.

20. Schwarz G. Estimating the dimension of a model. Ann Stat. 1978;6(2):461-4.

21. Nylund KL, Asparouhov T, Muthén BO. Deciding on the number of classes in latent class analysis and growth mixture modeling: a Monte Carlo simulation study. Struct Equ Model Multidiscip J. 2007;14(4):535-69.

22. Tein J-Y, Coxe S, Cham H. Statistical Power to Detect the Correct Number of Classes in Latent Profile Analysis. Struct Equ Modeling. 2013;20:640-57.

23. Heron J, Croudace TJ, Barker ED, Tilling K. A comparison of approaches for assessing covariate effects in latent class analysis. Longitudinal Life Course Stud. 2015;6(4):420-34

24. Sterne J, White J, Carlin J, Spratt M, Royston P, Kenward M, Wood A, Carpenter J. Multiple imputation for missing data in epidemiological and clinical research: potential and pitfalls. BMJ. 2009;338:b2393. 
25. Ramaswamy V, Desarbo WS, Reibstein DJ, Robinson WT. An empirical pooling approach for estimating marketing mix Elasticities with PIMS data. Mark Sci. 1993;12(1):103-24.

26. MacArthur GJ, Smith MC, Melotti R, Heron J, Macleod J, Hickman M, et al. Patterns of alcohol use and multiple risk behaviour by gender during early and late adolescence: the ALSPAC cohort. J Public Health. 2012;34(suppl_1): i20-30.

27. Evans H, Buck D. Tackling multiple unhealthy risk factors emerging lessons from practice. London: The King's Fund; 2018.

\section{Publisher's Note}

Springer Nature remains neutral with regard to jurisdictional claims in published maps and institutional affiliations.

Ready to submit your research? Choose BMC and benefit from:

- fast, convenient online submission

- thorough peer review by experienced researchers in your field

- rapid publication on acceptance

- support for research data, including large and complex data types

- gold Open Access which fosters wider collaboration and increased citations

- maximum visibility for your research: over $100 \mathrm{M}$ website views per year

At $B M C$, research is always in progress. 